Revista de MATEMÁticA: TeORÍA y APliCACIONEs 2016 23(2) : 321-338

CIMPA - UCR ISSN: 1409-2433 (PRINT), 2215-3373 (ONLINE)

\title{
ZEROES OF GENERALIZED FRESNEL COMPLEMENTARY INTEGRAL FUNCTIONS
}

\section{CEROS DE FUNCIONES INTEGRALES COMPLEMENTARIAS DE FRESNEL GENERALIZADAS}

JAIME LOBO-SEGURA* MARIO AlbERTO VILlaLOBOS-ARIAS ${ }^{\dagger}$

Received: 13 Feb 2015; Revised: 14 Apr 2016;

Accepted: 6 May 2016

*CIMPA, Universidad de Costa Rica, San José, Costa Rica. E-Mail: jaimelobosegura@ hotmail.com

${ }^{\dagger}$ Misma dirección que/Same address as: J. Lobo. E-Mail: mario.villalobos@ucr.ac.cr 


\begin{abstract}
Theoretical upper and lower bounds are established for zeroes of a parametric family of functions which are defined by integrals of the same type as the Fresnel complementary integral. Asymptotic properties for these bounds are obtained as well as monotony properties of the localization intervals. Given the value of the parameter an analytical-numerical procedure is deduced to enclose all zeros of a given function with an a priori error.
\end{abstract}

Keywords: Fresnel complementary integrals; zeroes of functions; theoretical bounds for zeroes; gamma distribution.

\title{
Resumen
}

Se establecen cotas superiores e inferiores teï $j$ 1/2ricas para los ceros de

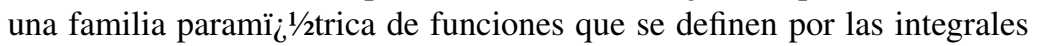
del tipo integral complementaria de Fresnel. Propiedades asintï $i^{1 / 2 t i c a s}$ de estas cotas se obtienen, asï $i^{1 / 2}$ como propiedades de monotonï $i^{1 / 2 a}$ de los intervalos de localizaciï $i^{1 / 2 n}$. Dado el valor del parï $i^{1 / 2}$ metro un proced-

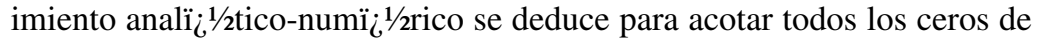
una funciï $\iota^{1 / 2 n}$ dada con un error a priori.

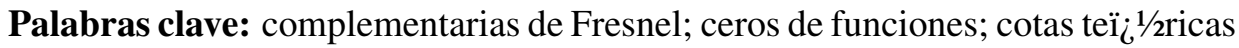
para ceros; distribuciï $i^{1 / 2 n}$ gamma.

Mathematics Subject Classification: 65H99, 33E20, 26A99.

\section{Introduction}

Consider the family of functions $s_{\alpha}, c_{\alpha}$ defined by a real parameter $\alpha, 0<\alpha$, with domain $\mathbb{R}_{+}$, as follows:

$$
s_{\alpha}(t)=\int_{t}^{\infty} \frac{\sin (u)}{u^{\alpha}} d u, \quad c_{\alpha}(t)=\int_{t}^{\infty} \frac{\cos (u)}{u^{\alpha}} d u, \quad t>0, \alpha>0 .
$$

It is well known that these integrals exist as improper Riemann integrals although they could be also interpreted as proper integrals in the theory of generalized Riemann integral. Among them the best known cases correspond to $\alpha=1,1 / 2$. For $\alpha=1$ the functions $s_{1}, c_{1}$ are generally denoted as $s i, c i$, the complementary of the integral sine and integral cosine respectively and are related to the integral sine and integral cosine functions, usually denoted by $S i$ and $\mathrm{Ci}$, the following way:

$$
S i(t)=\int_{0}^{t} \frac{\sin (u)}{u} d u, \quad s i(t)=\pi / 2-S i(t), \quad c i(t)=-C i(t) .
$$


Concerning $\alpha=1 / 2, s_{1 / 2}, c_{1 / 2}$ they correspond grosso modo to the complements of the well known Fresnel integrals

$$
S_{1 / 2}(t)=\int_{0}^{t} \frac{\sin (u)}{u^{1 / 2}} d u, C_{1 / 2}(t)=\int_{0}^{t} \frac{\cos (u)}{u^{1 / 2}} d u, \quad t>0
$$

(although these denominations are also used for the integrals $\left.1 / \sqrt{2 \pi} S_{1 / 2}\left(t^{2} \pi / 2\right), 1 / \sqrt{2 \pi} C_{1 / 2}\left(t^{2} \pi / 2\right)\right)$.

Many properties of functions $s_{1}, c_{1}, s_{1 / 2}, c_{1 / 2}$ are mentioned in [1] in sections 5.2, 7.3. From now on, the parametric family $s_{\alpha}, c_{\alpha}$ will be referred as generalized Fresnel complementary integral functions, and are denoted as FCI.

We study in this work the zeroes of the family FCI, that is the sets $\left\{s_{\alpha}=\right.$ $0\},\left\{c_{\alpha}=0\right\}$ for each value of the parameter $\alpha$. Some numerical experiments with these functions using different values of $\alpha$ allow us to make some conjectures about this problem. Some plots of these functions with the aid of Mathematica are shown below (see figures).

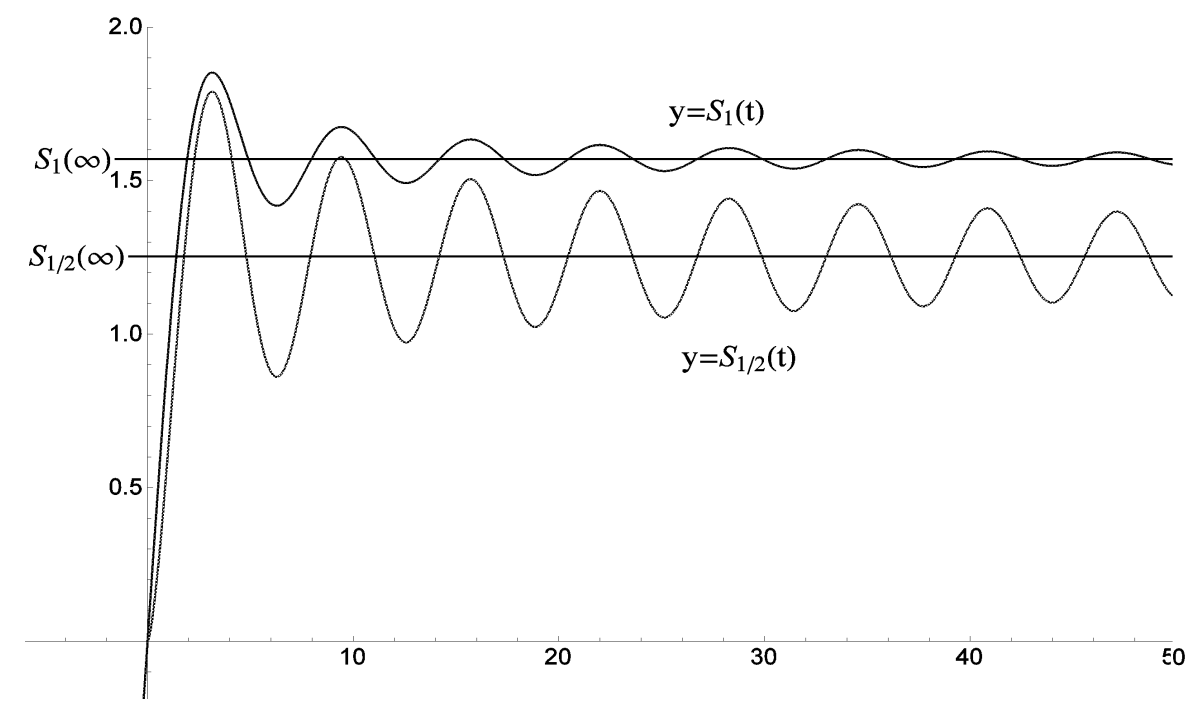

Figure 1: Graphs of $S_{1}(t), S_{1 / 2}(t)$.

It is noticed for example, that in each interval of monotonicity of the given FCI function there is a unique zero and that the set of zeroes is defined by an increasing sequence of reals which is very similar to an arithmetical one. In fact, some authors have found more accurate results of this kind for some particular cases of the parameter $\alpha$. A. E. Livingstone and L. Lorch in [8] and A. E. Livingstone in [7] studied the issue of zeroes of functions of type $t \rightarrow \int_{t}^{\infty} f(x) g(x) d x$ 


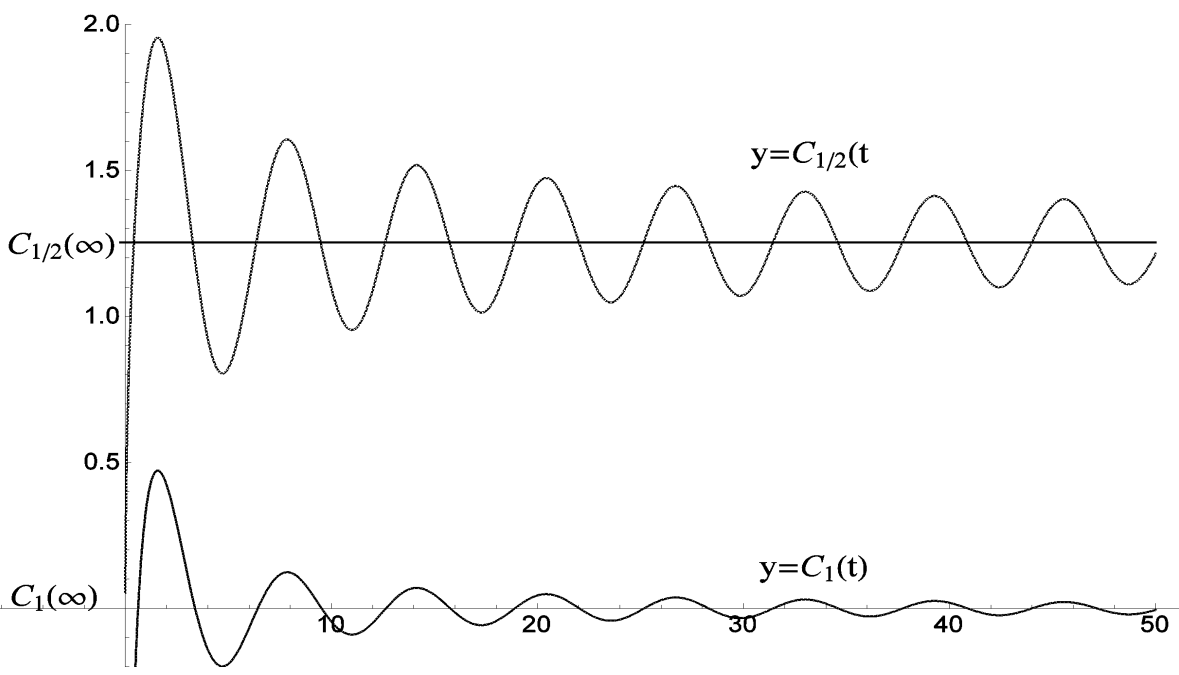

Figure 2: Graphs of $C_{1}(t), C_{1 / 2}(t)$.

under conditions including the function $s_{1}$ defined above. They proved that zeroes of a given function are actually an increasing sequence $c(n)$ adjacent to the sequence $n \pi+\pi / 2$ with $c(n)-(n \pi+\pi / 2) \downarrow 0$. However, their proof is not valid for $c_{1}$ because of the conditions imposed on $f$ and $g$. E. Kreyszig establishes in [5] some properties of functions $s_{1 / 2}, c_{1 / 2}$, the actual Fresnel complementary integrals, and approaches the issue of the approximation of zeros by establishing lower bounds and approximations based on asymptotical methods. However in all these works upper bounds for zeroes are unmentioned.

In [4] similar results for another family of function (a certain Lommel functions) is presented, where approximation intervals of fixed length are shown, and also a monotony property of the kind mentioned above.

The authors look for an extension of the results mentioned before to the whole family FCI for $\alpha>0$. It is shown that it is even possible to find theoretical upper bounds for the zeroes sought for and establish sharp asymptotic results for them as stated in the main theorem of the next section.

\section{Main theorem}

In the following theorem theoretical bounds are given in terms of approximation intervals for the zeros of the functions. 
Theorem 2.1. For every integer $n \geq 0$ and $\alpha>0$ let

$$
T(\alpha, n)=\frac{n \pi / 2+\sqrt{(n \pi / 2)^{2}+4 \alpha}}{2} .
$$

The zeros of $s_{\alpha}, c_{\alpha}$ all belong to the intervals of the form

$$
J(n)=(n \pi / 2, \min \{(n+1) \pi / 2, T(\alpha, n)\})
$$

( $n$ even in case of $c_{\alpha}, n$ odd in case of $s_{\alpha}$ ) and there is only one of them in each $J(n)$. Let $z(\alpha, n)$ be the only zero on $J(n)$. Then:

$$
z(\alpha, n)-n \pi / 2 \sim \frac{2 \alpha}{n \pi}, n \rightarrow \infty, \quad T(\alpha, n)-z(\alpha, n)=o\left(\frac{1}{n}\right), n \rightarrow \infty .
$$

The sequences with general term $z(\alpha, n)-n \pi / 2$ is strictly decreasing to zero.

Remark 2.2. The preceding result implies particularly that intervals of the form $[n \pi / 2,(n+1) \pi / 2], n$ integer, enclose zeroes of $s_{\alpha}, c_{\alpha}$. This generalizes Kreyszig's results ([5] lemma 1, pag. 503) where the case $\alpha=1 / 2$ is proven.

Remark 2.3. Since $T(\alpha, n)<\frac{2 \alpha}{n \pi}+\frac{n \pi}{2}, n \geq 1$ the right side of this inequality is also a theoretical upper bound of the zeros of index $n \geq 1$. In Kreyszig ([5] section 5) this value is proposed as an approximation of zeroes in case $\alpha=1 / 2$, but without proving that these are actually upper bounds. In this same work, many other approximations of zeroes are proposed, some of them being larger than $T(1 / 2, n)$ and so Theorem 2.1 is an improvement of the results in [5].

By means of the intervals $J(n)$ all zeros of $s_{\alpha}, c_{\alpha}$ can be enclosed with the peculiarity that their lengths decrease to zero as $n$ approaches infinity. This property will be used in conjunction with numerical methods to construct a simple procedure to enclose simultaneously all zeroes of a given function in FCI (section 5). One immediate consequence of this theorem is the possibility to find, as $T(\alpha, n) \leq(n+1) \pi / 2$ for every $n$, a sequence of enclosing intervals of the same length, taking as initial interval $J(0)$ whose length is $\sqrt{\alpha}$ :

Corollary 2.4. For $0<\alpha \leq \pi^{2} / 4$ the zeros of $s_{\alpha}, c_{\alpha}$ are enclosed in intervals

$$
(n \pi / 2, n \pi / 2+\sqrt{\alpha})
$$

$n \geq 0$, with $n$ odd in case $s_{\alpha}, n$ even in case $c_{\alpha}$.

As an example of corollary 2.4 enclosing intervals of length $1 / \sqrt{2}$ for each zero of $s_{1 / 2}, c_{1 / 2}$ are obtained. 


\section{Proof of the main theorem}

First, some preliminary results are established. The following is paramount and is related to the representation of the original functions by auxiliary ones.

Lemma 3.1. For $\alpha>0, t>0$ we have: $c_{\alpha}(t)+i s_{\alpha}(t)=e^{i t}\left(g_{\alpha}(t)+i f_{\alpha}(t)\right)$, where

$$
g_{\alpha}(t)=\frac{1}{\Gamma(\alpha)} \int_{0}^{\infty} \frac{\exp (-t y) y^{\alpha}}{1+y^{2}} d y, \quad f_{\alpha}(t)=\frac{1}{\Gamma(\alpha)} \int_{0}^{\infty} \frac{\exp (-t y) y^{\alpha-1}}{1+y^{2}} d y
$$

This representation is mentioned in Abramowitz \& Stegun [1] and in the NIST [10] for the case $\alpha=1$ ( formulae 5.2.8, 5.2.9, 5.2.12, 5.2.13 ) and $\alpha=$ $1 / 2$ ( formulae 7.3.5, 7.3.6, 7.4.25, 7.4.26). The case $0<\alpha<1$ is mentioned in Erdelyi ([3] pag. 150) under the form of asymptotic relations but not the representation itself by $g_{\alpha}, f_{\alpha}$. The authors give here a proof of this result in the general case $0<\alpha$ that resorts to an argument similar to Loya's ([9] theorem pag. 64), based on the Fubini theorem in the plane but simplified thanks to the Tonelli-Hobson theorem. Notice that integrals in the identity of lemma 3.1 are improper on the left hand and Lebesgue in the right hand. That allows to use the theory of Lebesgue Integral as we will see in the sequence.

As a first step, it is of primarily significance to interpreting the functions $g$, $f$ in the lemma in probabilistic terms. Let

$$
\mu_{\alpha, t}(d y)=\frac{t^{\alpha}}{\Gamma(\alpha)} e^{-t y} y^{\alpha-1} 1_{\{y>0\}} d y
$$

be the probability measure on $\mathbb{R}$ whose density with respect the Lebesgue measure is the gamma density function of parameters $\alpha, t$. It may be written:

$$
f_{\alpha}(t)=t^{-\alpha} \int \bar{f}(y) \mu_{\alpha, t}(d y), \quad g_{\alpha}(t)=t^{-\alpha} \int \bar{g}(y) \mu_{\alpha, t}(d y)
$$

where

$$
\bar{g}(y) \equiv \frac{y}{1+y^{2}}, \quad \bar{f}(y) \equiv \frac{1}{1+y^{2}} .
$$

Let us recall the identity:

$$
\frac{\Gamma(\alpha)}{t^{\alpha}}=\int_{0}^{\infty} \exp (-t y) y^{\alpha-1} d y
$$

Proof of lemma 3.1;

For each $0<t<A$ we define $F(t, A)$ as

$$
F(t, A)=\int_{t}^{A} \frac{\exp (i x)}{x^{\alpha}} d x
$$


For each $x>0$ we have

$$
x^{-\alpha}=\frac{1}{\Gamma(\alpha)} \int_{0}^{\infty} y^{\alpha-1} \exp (-x y) d y,
$$

and then:

$$
F(t, A)=\frac{1}{\Gamma(\alpha)} \int_{t}^{A} \int_{0}^{\infty} y^{\alpha-1} \exp (-x y+i x) d y d x
$$

The function $\left|y^{\alpha-1} \exp (-x y+i x)\right|=y^{\alpha-1} \exp (-x y)$ is integrable in $[t, A] \times$ $\mathbb{R}_{+}$,as the iterated integral

$$
\int_{t}^{A} \int_{0}^{\infty} y^{\alpha-1} \exp (-x y) d y d x=\Gamma(\alpha) \int_{t}^{A} x^{-\alpha} d x
$$

exists. The function $(x, y) \rightarrow y^{\alpha-1} \exp (-x y+i x)$ is therefore integrable in $[t, A] \times \mathbb{R}_{+}$( Tonelli-Hobson theorem) and by virtue of the Fubini theorem the order of integration may be reverted into (3.2) to get:

$$
F(t, A)=\frac{1}{\Gamma(\alpha)} \int_{0}^{\infty} y^{\alpha-1} \int_{t}^{A} \exp (-x y+i x) d x d y
$$

By denoting $G(x, y)=\frac{\exp (x(i-y))}{i-y}=-\exp (x(i-y)) \frac{(y+i)}{y^{2}+1}$ the interior integral is expressed as $G(A, y)-G(t, y)$ and replacing in 3.3 :

$$
F(t, A)=\frac{1}{\Gamma(\alpha)}\left(\int_{0}^{\infty} G(A, y) y^{\alpha-1} d y-\int_{0}^{\infty} G(t, y) y^{\alpha-1} d y\right)
$$

On the other hand, for every $A>t$, the modulus of the function $y \rightarrow$ $G(A, y) y^{\alpha-1}$ is bounded by the function $y \rightarrow y^{\alpha-1} \exp (-t y)\left|\frac{y+i}{y^{2}+1}\right|$, which is integrable in $[0, \infty]$. But in $(0, \infty)$ the pointwise convergence $G(A, y) y^{\alpha-1} \rightarrow 0$ occurs when $A \rightarrow \infty$ and so by the dominated convergence theorem it can be deduced that $\int_{0}^{\infty}\left(G(A, y) y^{\alpha-1}\right) d y \rightarrow 0$, when $A \rightarrow \infty$. Hence we obtain

$$
\lim _{A \rightarrow \infty} F(t, A)=1 / \Gamma(\alpha) \int_{0}^{\infty}\left(-G(t, y) y^{\alpha-1}\right) d y .
$$

The left member is $c_{\alpha}(t)+i s_{\alpha}(t)$, whereas the function under the integral sign in the right member is the sum of the functions $y \rightarrow \exp (t i) \exp (-t y) \frac{y^{\alpha}}{y^{2}+1}$ and $y \rightarrow i \exp (t i) \exp (-t y) \frac{y^{\alpha-1}}{y^{2}+1}$, which are both integrable in $[0, \infty)$ as their moduli are exponentially dominated in $+\infty$. The integral may be separated as the sum of the integrals of each term, and factorizing $\exp (t i)$ the lemma is proven. 
Remark 3.2. Formulae for $f_{\alpha}, g_{\alpha}$ in terms of parametric integrals can also be found in NIST (see [10], 8.21.4, 8.21.5). However, in contrast with lemma 3.1. these integrals are not Lebesgue integrals for all values of parameter $\alpha$.

For each integer $n \geq 0$ we denote: $I(n)=[n \pi / 2,(n+1) \pi / 2]$, where the functions $s_{\alpha}, c_{\alpha}$ are both monotone. The function $\theta_{\alpha}(t)$ is defined by

$$
\theta_{\alpha}(t)=\arctan \left(\frac{g_{\alpha}(t)}{f_{\alpha}(t)}\right)
$$

with domain $\mathbb{R}_{+}$, where $\arctan (x)=\int_{0}^{x} \frac{1}{1+u^{2}} d u$. Actually, the range of this function is included in $(0, \pi / 2)$ because $\theta_{\alpha}(t)=\pi / 2-\arg \left(g_{\alpha}(t)+i f_{\alpha}(t)\right)$ ( $\arg$ is the principal argument function), where $g_{\alpha}(t)+i f_{\alpha}(t)$ lies in the first quadrant.

The following gives a first enclosing of zeroes.

Corollary 3.3. a) Zeroes of $s_{\alpha}$ (resp. those of $c_{\alpha}$ ) lie in the interior of the intervals $I(n)$ for $n$ odd ( resp. in the interior of $I(n)$, $n$ even) and there is only one zero in each of them.

b) Zero in $I(n)$ defined in a) (of $s_{\alpha}$ or $c_{\alpha}$ depending of the parity of $n$ ) is the only fixed point of the function $\theta_{\alpha}+n \pi / 2$ in this interval.

Proof. a) For the existence and uniqueness of one zero it is enough to prove that each function $s_{\alpha}, c_{\alpha}$ changes sign exactly at the extrema of the intervals $I(n)$ for the $n$ having the same parity. Let us verify this for $s_{\alpha}$ : the relation of the preceding lemma applied at the points $n \pi / 2$ is written as:

$$
\begin{aligned}
& s_{\alpha}(n \pi / 2)=(-1)^{\llbracket n / 2 \rrbracket} f_{\alpha}(n \pi / 2), \text { if } n \text { is even, } \\
& s_{\alpha}(n \pi / 2)=(-1)^{\llbracket n / 2 \rrbracket} g_{\alpha}(n \pi / 2), \text { if } n \text { es odd, }
\end{aligned}
$$

$\left(\llbracket \cdot \rrbracket\right.$ designs the integer part of a given real number) and since $f_{\alpha}, g_{\alpha}$ are positive the result is proved.

For the functions $c_{\alpha}$ the reasoning is the same.

b) According to lemma 3.1

$$
\begin{aligned}
& c_{\alpha}(t)=\operatorname{Re}\left(e ^ { i t } \left(g_{\alpha}(t)+i f_{\alpha}(t)\right.\right. \\
& s_{\alpha}(t)=\operatorname{Im}\left(e ^ { i t } \left(g_{\alpha}(t)+i f_{\alpha}(t) .\right.\right.
\end{aligned}
$$

By calculating the expressions in the right hand and taking into account that functions $g_{\alpha}, f_{\alpha}$ never vanish, we get:

$$
s_{\alpha}(t)=0 \Leftrightarrow \cot (t)+\frac{g_{\alpha}(t)}{f_{\alpha}(t)}=0, c_{\alpha}(t)=0 \Leftrightarrow \tan (t)-\frac{g_{\alpha}(t)}{f_{\alpha}(t)}=0
$$


in the points where the cotangent and tangent functions are defined, which is true for every zero of $s_{\alpha}, c_{\alpha}$ since neither their sine or cosine vanishes. Let us verify the property of $s_{\alpha}$ 's zero within $I(2 n+1)$ : the cotangent function being invertible in the interior of $I=I(2 n+1)$ with inverse $\operatorname{arccot}+n \pi$, where $\operatorname{arccot}$ is the principal value of arcotangent function, and since the unique zero of $s_{\alpha}$ in $I$ is an interior point of this interval (according to a) above), the first equivalence above implies that this zero is a fixed point of $\operatorname{arccot}(-g / f)+n \pi$ in this interval. Besides $\operatorname{arccot}(-x)=\pi / 2+\arctan (x)$ and the assertion is proved in this case. The same procedure can be used for the zeroes of $c_{\alpha}$.

The intervals $I(n)$ constitute a first family of enclosing intervals for zeroes. In order to establish the intervals stated in the theorem we resort to the characterization of zeroes given in $b$ ) of corollary 3.3 as fixed points of the translations of the function $\theta_{\alpha}$. For this purpose, the authors denoted by $z(\alpha, n)$ the unique fixed point of $\theta_{\alpha}+n \pi / 2$ en $I(n)$, that is the only zero of the equation $\theta_{\alpha}(t)+n \pi / 2=t$ in the interval $I(n)$. Then, according to the preceding corollary, the set of zeroes we look for are:

$$
\left\{s_{\alpha}=0\right\}=\{z(\alpha, n), n \text { odd }\},\left\{c_{\alpha}=0\right\}=\{z(\alpha, n), n \text { even }\} .
$$

The following properties are of key importance to achieve the author's aim.

Lemma 3.4. For $0<\alpha \leq 1$ the following properties hold:

a) $\theta_{\alpha}$ is decreasing in $\mathbb{R}_{+}$,

b) $\theta_{\alpha}(t)<\arctan \left(\frac{\alpha}{t}\right), t>0$,

c) $\theta_{\alpha}(t) \sim \frac{\alpha}{t}, t \rightarrow \infty$

Proof. a) Since arctan is increasing in $\mathbb{R}_{+}$it is enough to prove that $t \rightarrow g_{\alpha}(t) / f_{\alpha}(t)$ is strictly decreasing in $\mathbb{R}_{+}$. The first derivative criterion is valid for this function since both $f_{\alpha}$ and $g_{\alpha}$ are derivable and $f_{\alpha}$ does not vanish. To determine the sign of the derivative, it was noticed that the rule for the derivation under the integral sign are applicable for $f_{\alpha}, g_{\alpha}$. Indeed to apply the classical rule of the Lebesgue theory [2], theorem 10.30, we only need to prove that the integrand function $H_{\alpha}(t, y)$ has partial derivative with respect $t$ for all $(t, y)$ and continuous for $t>0, y>0$ and bounded by an integrable function $\hat{H}_{\alpha}(y)$ defined for $t>a>0$; for that purpose take

$$
\hat{H}_{\alpha}(y)=\frac{y^{\alpha+1} e^{-a y}}{1+y^{2}} \text { for } g_{\alpha} \text { and } \hat{H}_{\alpha}(y)=\frac{y^{\alpha} e^{-a y}}{1+y^{2}} \text { for } f_{\alpha} .
$$


and thus obtaining:

$$
\begin{aligned}
\frac{d\left(g_{\alpha}(t)\right)}{d t} & =\frac{-1}{\Gamma(\alpha)} \int_{0}^{\infty}\left(\frac{e^{-t y} y^{\alpha+1}}{1+y^{2}}\right) d y \\
\frac{d\left(f_{\alpha}(t)\right)}{d t} & =\frac{-1}{\Gamma(\alpha)} \int_{0}^{\infty}\left(\frac{e^{-t y} y^{\alpha}}{1+y^{2}}\right) d y
\end{aligned}
$$

It is observed that $d\left(g_{\alpha}(t)\right) / d t=f_{\alpha}(t)-t^{-\alpha}, d\left(f_{\alpha}(t)\right) / d t=-g_{\alpha}(t)$, and applying the rule for the derivative of the quotient to $g_{\alpha}(t) / f_{\alpha}(t)$ the authors arrived at the following:

$$
\frac{d}{d t}\left(\frac{g_{\alpha}}{f_{\alpha}}\right)(t)=\frac{g_{\alpha}^{2}(t)+f_{\alpha}^{2}(t)-f_{\alpha}(t) t^{-\alpha}}{f_{\alpha}^{2}(t)} .
$$

Using the representation of $f_{\alpha}, g_{\alpha}$ in terms of the gamma distribution $\mu_{\alpha, t}(d y)$ ( see comments and notations for lemma 3.1), and resorting to the CauchySchwartz inequality to the integrals defining $f_{\alpha}, g_{\alpha}$ :

$$
f_{\alpha}^{2}(t)<t^{-2 \alpha} \int \bar{f}^{2}(y) \mu_{\alpha, t}(d y), \quad g_{\alpha}^{2}(t)<t^{-2 \alpha} \int \bar{g}^{2}(y) \mu_{\alpha, t}(d y)
$$

where the inequality is strict since $\bar{f}, \bar{g}$ are not constant, and then

$$
f_{\alpha}^{2}(t)+g_{\alpha}^{2}(t)<t^{-2 \alpha}\left(\int\left(\bar{f}^{2}(y)+\bar{g}^{2}(y)\right) \mu_{\alpha, t}(d y)\right)=t^{-\alpha} f_{\alpha}(t) .
$$

From this inequality and (3.5), it is deduced then that $d\left(g_{\alpha}(t) / f_{\alpha}(t)\right) / d t<0$ on $\mathbb{R}_{+}$, and thus the decreasing property is proved.

b) Resorting again to the representation of $f_{\alpha}, g_{\alpha}$ mentioned in a) we get

$$
\frac{g_{\alpha}(t)}{f_{\alpha}(t)}=\frac{\int \bar{g}(y) \mu_{\alpha, t}(d y)}{\int \bar{f}(y) \mu_{\alpha, t}(d y)} .
$$

On the other side $\int \bar{g}(y) \mu_{\alpha, t}(d y)=\int y \bar{f}(y) \mu_{\alpha, t}(d y)$, and since the identity function in $\mathbb{R}$ is increasing while $y \rightarrow \bar{f}(y)$ is decreasing in the support of the measure $\mu_{\alpha, t}(d y)$, as a consequence of Harris inequality (see for example theorem 5-13, chap. IV in Lindvall) we get

$$
\int \bar{g}(y) \mu_{\alpha, t}(d y) \leq \int y \mu_{\alpha, t}(d y) \int \bar{f}(y) \mu_{\alpha, t}(d y)
$$

and in conjunction with the above identity we get $g_{\alpha}(t) / f_{\alpha}(t) \leq \int y \mu_{\alpha, t}(d y)$. But the right member of this inequality is the mean of the distribution $\mu_{\alpha, t}(d y)$ 
whose value is $\frac{\alpha}{t}$. Therefore $\arctan \left(g_{\alpha}(t) / f_{\alpha}(t)\right) \leq \arctan (\alpha / t)$, as we wished.

c) Since arctan is equivalent to the identity function in 0 it is enough to prove that $t \rightarrow g_{\alpha}(t) / f_{\alpha}(t)$ is equivalent to $\alpha / t$ in $+\infty$. Now then, $g_{\alpha}, f_{\alpha}$ are respectively the Laplace transforms of the functions $G_{\alpha}, \quad F_{\alpha}$, with $G_{\alpha}(y) \sim$ $y^{\alpha} / \Gamma(\alpha), F_{\alpha}(y) \sim y^{\alpha-1} / \Gamma(\alpha) y \rightarrow 0$. According to Watson lemma $g_{\alpha}(t) \sim$ $\Gamma(\alpha+1) / \Gamma(\alpha) t^{\alpha+1}=\alpha / t^{\alpha+1}, f_{\alpha}(t) \sim 1 / t^{\alpha}$ when $t \rightarrow \infty$ and therefore $g_{\alpha}(t) / f_{\alpha}(t) \sim \alpha / t$ when $t \rightarrow \infty$, thus this being the author s conclusion.

Summarizing the previous results we get:

End of the proof of theorem 2.1: From a) of corollary 3.3 to obtain the upper bound stated in the theorem it is sufficient to prove that $T(\alpha, n)$ is an upper bound of $z(\alpha, n)$. From the inequality $\theta_{\alpha}(t)<\frac{\alpha}{t}$ ( b) of lemma 3.4 and the fact that $\arctan (x)<x$ in $\left.\mathbb{R}_{+}\right)$it is deduced that the fixed point of $\theta_{\alpha}(t)+n \pi / 2$ is lower than the correspondent of the function $t \rightarrow \frac{\alpha}{t}+n \pi / 2$ in $\mathbb{R}$. This last one can be calculated exactly by solving the equation $\frac{\alpha}{t}+n \pi / 2=t$, equivalent to a quadratic polynomial equation whose only root superior to $n \pi / 2$ corresponds to the value $T(\alpha, n)$ declared in the statement. The conclusion follows from $\mathrm{b}$ ) of corollary 3.3

Let us prove the other properties.

\section{Proof. a) Asymptotic properties:}

To analyze the behavior of $z(\alpha, n)-n \pi / 2, n \rightarrow \infty$ let us observe first that since $z(\alpha, n)$ diverges to $+\infty$, from c) of lemma 3.4 it is deduced that $\theta_{\alpha}(z(\alpha, n)) \sim \alpha / z(\alpha, n), n \rightarrow \infty$ and by the fixed point property of corollary 3.3 it is obtained $z(\alpha, n)-n \pi / 2 \sim \alpha / z(\alpha, n)$. Since $1 / z(\alpha, n) \sim 2 / n \pi$ as $n \rightarrow \infty$, it is finally deduced that $z(\alpha, n)-n \pi / 2 \sim 2 \alpha / n \pi, n \rightarrow \infty$.

For $T(\alpha, n)-z(\alpha, n), n \rightarrow \infty$ we write

$$
T(\alpha, n)-z(\alpha, n)=(T(\alpha, n)-n \pi / 2)-(z(\alpha, n)-n \pi / 2) .
$$

Writing now $T(\alpha, n)$ as $n \pi / 4\left(1+\sqrt{1+4 \alpha /(n \pi / 2)^{2}}\right)$ and applying a second order Taylor expansion around 0 of $x \rightarrow \sqrt{1+x^{2}}$ we obtain $T(\alpha, n)=n \pi / 2+2 \alpha / n \pi+o(1 / n)$. Then $(T(\alpha, n)-n \pi / 2)=2 \alpha / n \pi+o(1 / n)$, and since it was proved that $z(\alpha, n)-n \pi / 2 \sim 2 \alpha / n \pi$, when replacing in equation (3.6) and substracting the asymptotic formulae the term $2 \alpha / n \pi$ is eliminated remaining only the sum of terms of the form $o(1 / n)$, which is the sought relation. 


\section{b) Growth and convergence properties:}

The convergence to 0 is a consequence of the asymptotic properties. To establish these ones let us first consider the case $(z(\alpha, n)-n \pi / 2, n \in \mathbb{N})$ : given an integer $n$, from $\mathrm{b}$ ) of corollary 3.3 we know that $z(\alpha, n)=\theta_{\alpha}(z(\alpha, n))+$ $n \pi / 2$, and on the other hand $\theta_{\alpha}(z(\alpha, n+1))<\theta_{\alpha}(z(\alpha, n))$ by virtue of the monotony property of $\theta_{\alpha}$ ( a) from lemma 3.4). Then $z(\alpha, n)+\pi / 2>z(\alpha, n+$ $1)$, which is equivalent to $z(\alpha, n)-n \pi / 2>z(\alpha, n+1)-(n+1) \pi / 2$.

\section{An improved lower bound}

The sequence of lower bounds stated in the theorem 2.1, $n \pi / 2$, can be improved in such a way that the the sequence of errors is asymptotically as good as that of the upper bounds. Let $I(n)=[n \pi / 2,(n+1) \pi / 2]$, and let $i n t(I(n))$ be the interior of $I(n)$.

Theorem 4.1. Let $N$ be the integer such that for $n \geq N$ the inequality ( $n+$ 1) $\pi / 2>T(\alpha, n)$ holds. Let $h$ be the function defined in $\bigcup_{n \geq N}$ int $(I(n))$ by

$$
h(t)= \begin{cases}c_{\alpha}(t) & \text { if } t \in \operatorname{int}(I(n)) \text { and } n \text { even, } \\ s_{\alpha}(t) & \text { ift } \in \operatorname{int}(I(n)) \text { and } n \text { odd. }\end{cases}
$$

Then for every $n \geq N$ the real real $\tau(\alpha, n)$ defined by:

$$
\tau(\alpha, n)=T(\alpha, n)-\left(h^{\prime}\right)^{-1}(T(\alpha, n)) h(T(\alpha, n))
$$

is well defined and is a lower bound of the zero $z(\alpha, n)$ in $I(n)$. Besides the following relation holds:

$$
\tau(\alpha, n)-z(\alpha, n)=o\left(\frac{1}{n}\right), n \rightarrow \infty .
$$

\section{Proof. a) Bound property}

First it is proved that $\tau(\alpha,$.$) are actually lower bounds.$

Let us consider an $n \geq N$, that we suppose even. Let us prove that $\tau(\alpha, n)$ is a lower bound of the zero of $c_{\alpha}$ located in $I(n)$. Since $T(\alpha, n)$ belongs to $\operatorname{int}(I(n))$ their cosine is not 0 , and then $\tau(\alpha, n)$ is well defined. We observe that the real $\tau(\alpha, n)$ is the abscissa of the point of intersection of the tangent line to $y=c_{\alpha}(x)$ and the abscissa axis, that is the value of the first iteration of the Newton method applied to the function $c_{\alpha}$ starting at $T(\alpha, n)$. To verify that $\tau(\alpha, n)$ is actually a lower bound it is sufficient to prove that function $c_{\alpha}$ is convex in $I(n)$ whenever is decreasing in $I(n)$, and concave whenever is increasing 
in $I(n)$. In order to reach this point the sign of the first and second derivatives of $c_{\alpha}$ are analyzed:

$$
c_{\alpha}^{\prime}(t)=-\frac{\cos (t)}{t^{\alpha}}, \quad c_{\alpha}^{\prime \prime}(t)=t^{-\alpha-1}(t \sin (t)+\alpha \cos (t)) .
$$

The derivative $c_{\alpha}^{\prime}$ is negative in $I(n)\left(c_{\alpha}\right.$ is decreasing in $\left.I(n)\right)$ only if the cosine function is positive in this interval. But the cosine and sine functions have the same sign in $I(n)$ because $n$ is even, and therefore from the above expression for $c_{\alpha}^{\prime \prime}$ it is deduced that the second derivative is positive. Then $c_{\alpha}$ is convex in $I(n)$. The case when $c_{\alpha}$ is increasing in $I(n)$ is alike.

The case $n \geq N$ and $n$ is odd is similar to the last one. In this case the problem is about a lower bound for the zero of $s_{\alpha}$ in $I(n)$, that holds if we prove as before that $s_{\alpha}$ is convex in $I(n)$ whenever is decreasing in $I(n)$, concave whenever is increasing. Indeed, when the function is convex and decreasing in $I(n)$, the tangent line of the function at $T(\alpha, n)$ is below the graph of the original function and with negative slope and then its intersection with the abscissa axis, $\tau(\alpha, n)$, is at the left of the zero $z(\alpha, n)$. For the case concave and increasing the reasoning is similar.

In order to reach to this the calculation of derivatives is considered:

$$
s_{\alpha}^{\prime}(t)=-\frac{\sin (t)}{t^{\alpha}}, \quad s_{\alpha}^{\prime \prime}(t)=-t^{-\alpha-1}(t \cos (t)-\alpha \sin (t))
$$

it is concluded by recalling that the sine and cosine functions are of different sign in $I(n)$ when $n$ is odd.

\section{b) Asymptotic property:}

Consider the case of an even integer $\mathrm{n}$ and $n \geq N$ and the reals $\tau(\alpha, n), z(\alpha, n)$ associated to it. It is obtained, recalling that $c_{\alpha}^{\prime}(t)=-\cos (t) / t^{\alpha}$ :

$$
\tau(\alpha, n)=T(\alpha, n)+\left(\cos (T(\alpha, n)) / T(\alpha, n)^{\alpha}\right)^{-1} c_{\alpha}(T(\alpha, n)) .
$$

Using a first order Taylor expansion of $c(\alpha, n)$ at $z(\alpha, n)$ it is obtained:

$$
c_{\alpha}(T(\alpha, n))=-\cos (w(\alpha, n)) / w(\alpha, n)^{\alpha}(T(\alpha, n)-z(\alpha, n))
$$

where $w(\alpha, n)$ is a real number between $z(\alpha, n)$ and $T(\alpha, n)$. Hence

$$
\begin{aligned}
& \tau(\alpha, n)-T(\alpha, n) \\
& \quad=-(\cos (w(\alpha, n)) / \cos (T(\alpha, n)))(T(\alpha, n) / w(\alpha, n))^{\alpha}(T(\alpha, n)-z(\alpha, n)) .
\end{aligned}
$$

But $(w(\alpha, n), n \geq N),(T(\alpha, n), n \geq N)$ are sequences adjacent to the sequence $(n \pi / 2, n \geq N)$ with $|\cos (n \pi / 2)|=1$, and by virtue of the uniform 
continuity of the cosine function in $\mathbb{R}$ the quotient $\cos (w(\alpha, n)) / \cos (T(\alpha, n))$ converges to 1 as $n \rightarrow \infty$. On the other side the quotient $w(\alpha, n) / T(\alpha, n)$ converges to 1 as $n \rightarrow \infty$ and from the expression stated above it is concluded that $|\tau(\alpha, n)-T(\alpha, n)| \sim|z(\alpha, n)-T(\alpha, n)|$ as $n \rightarrow \infty$.

Thanks to theorem $2.1 \tau(\alpha, n)-T(\alpha, n)=o(1 / n)$ is met as $n \rightarrow \infty$ and $n$ is even, whence it is concluded that $\tau(\alpha, n)-z(\alpha, n)=o(1 / n)$ as $n \rightarrow \infty$ and $n$ is even, since $|\tau(\alpha, n)-T(\alpha, n)|>|z(\alpha, n)-\tau(\alpha, n)|$.

The case of odd $n$ is treated similarly as the previous one.

Remark 4.2. It is seen from the proof of the theorem above that if $\tau(\alpha, n)$ is defined from any other real number in $\operatorname{int}(I(n))$ instead of $T(\alpha, n)$ a lower bound of $z(\alpha, n)$ is obtained as well. In particular, this is true for $n \pi / 2$, but then an improvement of the asymptotic quality with respect to $T(\alpha, n)$ is not guaranteed. In fact, retracing the proof of theorem it can be asserted that $|\tau(\alpha, n)-n \pi / 2| \sim$ $|z(\alpha, n)-n \pi / 2|$, and best to be concluded is that $\tau(\alpha, n)-z(\alpha, n)=O(1 / n)$.

Remark 4.3. It cannot be ensured that $\tau(\alpha, n)$ is larger than $n \pi / 2$ for all the integers $n$. However the asymptotic property of the sequence of the $\tau(\alpha, n)$ together with the result of theorem 2.1 proves that $\tau(\alpha, n)>n \pi / 2$ from a certain value $n$.

\section{Numerical examples}

As a numerical test of the bounds found in theorems 2.1 and 4.1 , the case of parameter $\alpha=1$ is considered, and numerical approximations of $\operatorname{si}(t), c i(t)$ zeroes are compared with the bounds values. This issue being equivalent to that of finding zeroes of $S i(x)-\pi / 2$ and $C i(x)$, the Newton method is applied to these functions in different intervals $I(n)$, and the iterations are then of the form:

$$
x_{k+1}=x_{k}\left(1-\frac{\operatorname{Si}\left(x_{k}\right)-\pi / 2}{\sin \left(x_{k}\right)}\right), \quad x_{k+1}=x_{k}\left(1-\frac{C i\left(x_{k}\right)}{\cos \left(x_{k}\right)}\right) .
$$

Here $x_{0}=n \pi / 2$ is taken as initial value in 5.1. The procedure 5.1 is programmed in Mathematica, taking advantage of the predefined functions "SinIntegral[ $t]$ " and "CosIntegral[ $t]$ " for $S i(t), C i(t)$ in this package. The calculations are stopped when $\left|x_{k}-x_{k-1}\right|<10^{-15}$ is reached, obtaining approximations $\bar{z}(1, n)$ for $z(1, n)$. For some particular values of $n$ the comparisons with the theoretical bounds are shown in Table 1 . 
Table 1: Approximations of zeroes and theoretical bounds for $n$.

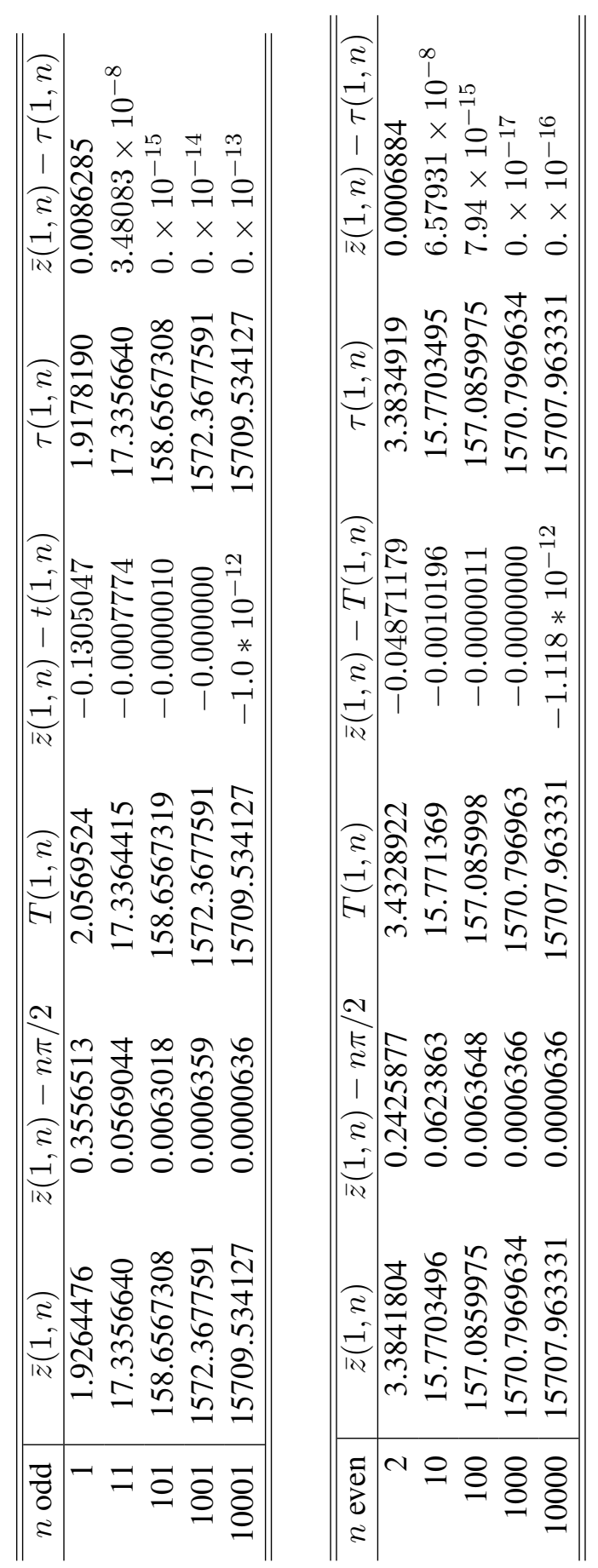

Rev.Mate.Teor.Aplic. (ISSN print: 1409-2433; online: 2215-3373) Vol. 23(2): 321-338, July 2016 
It is readily seen that in these cases, for large values of $n, z(1, n)$ is closer to $T(1, n)$ than $n \pi / 2$, which is consistent with theorem 2.1, and on the other hand that $\tau(1, n)$ is a better approximation than $T(1, n)$, confirming theorem 4.1. Besides, the error associated to $\tau(1, n)$ seems to be the square of the error associated to $T(1, n)$, confirming once again the quality of the bounds $\tau(1, n)$.

Now then, if enclosing intervals of predetermined length for the zeroes of $s_{\alpha}, c_{\alpha}$ are looked for, first an priori error $\epsilon>0$ is chosen; it is easy verified that for $n>\frac{2 \alpha}{\pi \varepsilon}$ the length of $J(n)=\min (T(\alpha, n),(n+1) \pi / 2)-n \pi / 2$ is less than $\varepsilon$, the lengths of all $J(m), m \geq n$ being also less than $\varepsilon$ according to theorem 2.1. For $s_{\alpha}, c_{\alpha}$ s zeroes lying in $J(m), m<n$, a numerical procedure is applied in order to obtain enclosing intervals of the sought degree of approximation. In case $\alpha=1$ if $\varepsilon=10^{-1}$ then for $n \geq 7$ the length of $J(m)<10^{-1}$. For zeroes $z(1,0), z(1,1), \ldots, z(1,6)$ numerical methods are used. It is useful to notice that for $n=2,3,4,5,6$ the values $\tau(1, n), T(1, n)$ can be taken as extrema of localization intervals, whereas for $n=0,1$ the Newton method is applied. The localization intervals are shown in Table 2 (where $a(1, n), b(1, n)$ denote the left and right extremum respectively ).

\begin{tabular}{c|cccl}
\hline \hline$n$ & $\bar{z}(1, n)$ & $a(1, n)$ & $b(1, n)$ & $b(1, n)-a(1, n)$ \\
\hline 0 & 0.6165054856 & 0.6100000000 & 0.630000000 & 0.0200000 \\
1 & 1.9264476603 & 1.9178190733 & 1.940000000 & 0.022181 \\
2 & 3.3841804226 & 3.3834919312 & 3.432892216 & 0.049400 \\
3 & 4.8938359526 & 4.8937384827 & 4.915814086 & 0.02207560 \\
4 & 6.4270477441 & 6.4270275142 & 6.438500963 & 0.01147345 \\
5 & 7.9726826200 & 7.9726771577 & 7.979305819 & 0.006629 \\
6 & 9.5255754576 & 9.5255736692 & 9.529712916 & 0.004139 \\
\hline$>6$ & & $J(n)$ & & $<10^{-1}$ \\
\hline \hline
\end{tabular}

Table 2: Approximation intervals of zeroes with error less than $10^{-1}$.

Similar numerical experiments can be performed in the case $\alpha=1 / 2$, since Mathematica has programmed functions for the Fresnel integrals.

\section{Conclusions and future work}

In this work we have found theoretical bounds of the zeros of the parametric family of functions FCI presented in theorems 2.1. and 4.1. These theoretical bounds were proven by means of numerical calculations presented in the tables of the previous section. 
We could improve the bounds found here for example by attempting to reduce the order of approximation error from $o(1 / n)$ to $o\left(1 / n^{p}\right), p>1$. On the other hand it would be important to know whether the property of monotony of theorem 2.1, is valid for the sequence $(T(\alpha, n)-\tau(\alpha, n) ; n \geq 1)$, which would allow the improvement of the simultaneous approximation procedure for the zeroes described in section 5 .

\section{Acknowledgment}

We wish to thank the anonymous referees for their valuable comments and suggestions.

\section{References}

[1] Abramowitz, M.; Stegun, I.A. (1964) Handbook of Mathematical Functions. National Bureau of Standards, Applied Mathematics Series 55, Washington DC.

[2] Apostol, T. (1975) Mathematical Analysis. Addison Wesley, USA.

[3] Erdï ¿ำlyi, A.; Magnus, W.; Oberhettinger, F.; Tricomi, F.G. (Eds.) (1953) Higher Transcendental Functions, Volume 1. 1. McGraw-Hill, New York.

[4] Koumandos, S.; Lamprecht, M. (2012) "The zeros of certain Lommel functions", Proceedings of The American Mathematical Society 140(9): 30913100 .

[5] Kreyszig, E. (1957) "On the complementary functions of the Fresnel integrals", Canadian Journal of Mathematics 9(4): 500-510.

[6] Lindvall, T. (2002) Lectures on the Coupling Method. Dover, New York.

[7] Livingstone, A.E. (1954) "The zeros of a certain class of indefinite integrals", Proceedings of the American Mathematical Society 5(2): 296-300.

[8] Livingstone, A.E.; Lorch, L. (1956) "The zeros of certain sine-like integrals", Proceedings of the American Mathematical Society 7(5): 813-816.

[9] Loya, P. (2005) "Dirichlet and Fresnel integrals via iterated integration", Mathematics Magazine 78(1): 63-67. 
[10] NIST Digital Library of Mathematical Functions (2016) "8.21 Generalized Sine and Cosine Integrals", http://dlmf.nist.gov/8.21, consulted: Jannuary 25, 2016. 